\title{
Motivational deficits after brain injury: effects of bromocriptine in 11 patients
}

\author{
J H Powell, S Al-Adawi, J Morgan, R J Greenwood
}

\begin{abstract}
Objective-To test the hypothesis that treatment with bromocriptine would ameliorate deficits in clinical motivation, responsiveness to reward, and frontal cognitive function after brain injury.

Method-An open trial in six men and five women who had had either traumatic brain injury or subarachnoid haemorrhage between two months and five years previously. After repeated baseline assessments, bromocriptine was given in gradually increasing doses. Assessments were repeated at increasing doses, during maintenance, and after withdrawal. Novel structured instruments for quantifying motivation were developed; measures of anxiety and depression, and cognitive tests sensitive to motivation or frontal lobe involvement were also given.

Results-Bromocriptine treatment was followed by improved scores on all measures other than mood. Improvement was maintained after bromocriptine withdrawal in eight of the patients.

Conclusion-Poor motivation in patients with brain injury may result from dysfunction in the mesolimbic/mesocortical dopaminergic circuitry, giving rise to associated deficiencies in reward responsiveness and frontal cognitive function.
\end{abstract}

$(\Im$ Neurol Neurosurg Psychiatry 1996;60:416-421)

Department of

Psychology,

Goldsmiths College,

Lewisham Way,

New Cross, London

SE14 6NW, UK

J H Powell

Regional Neurological

Rehabilitation Unit,

Homerton Hospital,

Homerton Row,

London E9 6SR,UK

$\mathrm{J} H$ Powell

$S$ Al-Adawi

J Morgan

R J Greenwood

Department of

Psychology, Institute

of Psychiatry, De

Crespigny Park,

Denmark Hill, London

SE5 8AF, UK

$S$ Al-Adawi

Correspondence to:

Dr Jane Powell, Department

of Psychology, Goldsmiths

Oof Psychology, Goldsmith

College, Lewisham Way,

Received 21 August 1995

and in revised form

14 December 1995

Accepted 22 December 1995
Keywords: motivation; abulia; dopamine; bromocriptine

Of the many behavioural problems that can follow brain injury, passivity and loss of drive (abulia) must rank among the most profoundly debilitating and intractable. A pervasive failure to initiate activities spontaneously or to respond to encouragement and prompting can not only result in social alienation but also impede rehabilitation. Behaviour modification techniques are of documented success in reducing the frequency of overtly disruptive behaviours ${ }^{1}$ but there is far less evidence of their success in increasing levels of effortful behaviour. Indeed, a recent follow up study ${ }^{2}$ has confirmed that poor psychosocial outcome a year after brain injury is predicted by initial impairment on cognition and energy items in the neurobehavioural rating scale. ${ }^{3}$

By contrast, there have recently been reports that such motivational deficits respond well to treatment with drugs which have dopaminergic effects. ${ }^{4-10}$ Most of these reports consist of anecdotal descriptions of single cases or series of single cases, and it is not clear, for example, whether similar unreported cases have failed to respond to treatment. However, in the light of growing evidence from both animal and human research implicating the mesolimbic and mesocortical dopamine system in normal motivation, a clear theoretical basis for the efficacy of such treatment can be constructed, and the preliminary clinical findings therefore take on a greater relevance.

The dopaminergic pathways implicated in motivation originate in the ventral tegmentum, and project to the nucleus accumbens and medial/sulcal prefrontal cortex ${ }^{1112}$; there are reciprocal glutamatergic projections from the frontal cortex to the nucleus accumbens via the entorhinal cortex. An extensive scientific literature suggests that dopamine release in the nucleus accumbens or ventral tegmentum may underlie the instantaneous experience of pleasure elicited by potent reinforcers such as opiate and stimulant drugs, ${ }^{13} 14$ electrostimulation, ${ }^{15}{ }^{16}$ food and water, ${ }^{17} 18$ and sex. ${ }^{18}$ The nigrostriatal dopamine system may also be implicated in rewarding brain stimulation. ${ }^{19}$ However, there is also evidence that dopamine is released in response to aversive stimulation, ${ }^{20}$ and one current view ${ }^{21}$ is that dopamine release mediates the behavioural response to motivationally relevant stimuli rather than the experience of reward itself.

If organic brain injury disrupted dopamine transmission within this system, a predictable consequence would be a failure to respond to normally motivating events, due either to reduced capacity for the experience of pleasure or to reduced ability to respond to available rewards with the requisite behavioural output. In either case, patients should show reduced levels of goal directed behaviour. Consistent with this model, impairments of effortful behaviour do characterise several neuropsychiatric conditions in which there is evidence of dysfunction in mesolimbic/ mesocortical dopamine systems-namely, Parkinson's disease, ${ }^{22}$ negative type schizophrenia, ${ }^{23}$ and major depression. ${ }^{24}$ In all three conditions dopamine agonists have had some success in enhancing activity levels. ${ }^{22} 25-30$ Whereas the organic basis of these neuropsychiatric conditions remains to some extent speculative, there is extensive evidence that focal lesions to the frontal cortex, innervated by mesocortical dopamine projections, can indeed lead to cognitive and behavioural deficits consistent with the dopaminergic model of motivation. Damage to the dorsolat- 
eral frontal cortex can give rise to a "pseudodepressive" syndrome ${ }^{31}$ characterised by passivity and flattened affect, reduced verbal output, and a slowness to initiate or respond. Cognitively, the frontal cortex seems to be critically involved in "executive" functions including the generation and monitoring of strategic action, ${ }^{32}$ functions essential to complex goal directed behaviour.

The present study was designed both to evaluate systematically the effectiveness of treatment with a dopamine agonist (bromocriptine) in alleviating poor motivation in a consecutive series of patients with organic brain injury, and to test predictions derived from the theoretical model described. Specifically, it was hypothesised that any clinical improvements in effortful behaviour should be paralleled by increasing responsiveness to incentives in an experimental setting, and by improvements in indices of frontal cognitive function.

\section{Design}

The study was conducted with patients receiving rehabilitation after single incident brain injury at the Regional Neurological Rehabilitation Unit (RNRU) of Homerton Hospital. Patients identified clinically as manifesting poor motivation, which was not obviously secondary to low mood and which resulted in pervasive passivity both in treatment and in their daily lives, were routinely considered for treatment with bromocriptine, a postsynaptic dopamine agonist with a particular affinity for D2 receptors.

A series of 10 consecutive patients from the RNRU, and one additional patient treated at another hospital, all receiving bromocriptine treatment, were assessed with identical single case methodology. Assessments were conducted twice before the start of treatment, across a period of 14-21 days, as a repeated baseline to establish that their functioning was stable. Bromocriptine was then introduced, using the regime described below, and assessments were repeated after every $2.5 \mathrm{mg}$ increment. If and when improvements were noted the dose was stabilised and the assessment was repeated one week later. If the improvement was maintained, bromocriptine was withdrawn and patients were reassessed on two further occasions, after a minimum of two weeks. Any patients whose gains reversed were to have the drug re-introduced, with further assessments to determine whether improvements were re-instated. In the event, no patient had a second phase of treatment, for reasons which will become apparent.

Informed consent to the assessments was gained on every assessment occasion, and any tests that patients were unwilling to complete were terminated.

DRUG REGIME

Bromocriptine is a postsynaptic dopamine agonist with a particular affinity for D2 receptors. It is widely used in the treatment of Parkinson's disease and hyperprolacti- naemia. ${ }^{33}$ Although it can produce nausea and gastric symptoms in some patients, these can in most cases be effectively ameliorated by concurrent prescription of domperidone. It can cause first dose or dose dependent hypotension, and there are accordingly some medical contraindications to its use, particularly ischaemic heart disease. No patient with such disease or a history of psychosis was offered the treatment. While they were taking bromocriptine, the patients' blood pressure was monitored over the first week, and any adverse gastric effects were noted. The starting dose was $2.5 \mathrm{mg} / \mathrm{day}$, and this was increased by $2.5 \mathrm{mg} /$ day per week to a maximum of 10 $\mathrm{mg} /$ day.

None of the 11 patients described here showed any adverse side effects resulting either in premature discontinuation of bromocriptine or additional medication. However, one additional patient was started on bromocriptine but withdrew after suffering nausea on the first day. He refused the option of restarting with concurrent domperidone.

\section{PATIENTS}

Six men and five women participated. Their ages ranged between 26 and 55 (mean 36) years. Eight had sustained traumatic head injury and three (all women) subarachnoid haemorrhage. Neuroimaging data (usually CT) disclosed focal right sided damage in five patients, left sided in one, and bilateral or diffuse damage in five. Focal damage to the frontal lobes was noted in three patients. Time elapsed since brain injury ranged between two months and five years. For three patients it was less than six months, for five it was between six and 15 months, and for three it exceeded two years.

Eight patients started bromocriptine treatment while inpatients; one transferred to another hospital midtrial but continued treatment and assessments there. Two were discharged immediately after bromocriptine withdrawal, so the two postwithdrawal assessments were conducted in their own homes. The remaining three patients were treated in the community throughout, with their general practitioners prescribing.

Where possible, all of the measures described below were given to all patients on each assessment occasion. However, in some cases certain of the tests could not be used; reasons for this are given.

\section{MEASURES}

\section{Assessment of motivation: therapy participation}

For the eight inpatients, all of their therapists kept a structured record of behaviour during each session within the week of the assessment:

Percentage participation index (PPI)Therapists recorded the number of minutes (a) of direct contact with the patient and (b) for which they judged the patient to have been actively participating; (b) was computed as a percentage of $(a)$ to give the PPI. In a related study with 54 patients admitted to the RNRU (Al-Adawi, Powell, and Greenwood, unpub- 
lished observations), this measure was normally distributed and had high interrater reliability, with correlations of between 0.79 and 0.90 for scores given by different therapists treating the same patient in different sessions within the same week $(P<0.001$ for every pair of raters). Ratings were also very stable, with correlations between different sessions conducted by the same therapist exceeding 0.90 $(P<0.001)$.

Prompting/spontaneity-Therapists rated the level of prompting given, with a 5 point scale $(0=$ none, $4=$ constant $)$. To simplify comparisons with other measures, so that a low score indicates a passive state, ratings have been reverse keyed to index "spontaneity".

Motivation-Therapists rated patients' perceived level of motivation during each session on a 5 point scale ranging from 0 (extremely low) to 4 (extremely high).

For each of the above indices, an average was computed from as many as possible of the sessions conducted within the week. The number of treatment sessions ranged between two and five (median three). These data were complete across all five assessment occasions for six patients. The remaining five patients were living in the community at some or all of the assessment points.

However, it was predicted that level of motivation should also affect performance on cognitive tasks, and administration of these was unaffected by inpatient or outpatient status.

Responsiveness to experimental incentive: the CARROT

The card arranging reward responsivity objective test (CARROT) was devised specially for the present research, and measures the extent to which patients increase their speed of performance on a simple psychomotor task ${ }^{34} 35$ when offered a small financial incentive. It involves within subject comparisons to assess individual responsiveness to incentive.

Briefly, the subject is presented with a stack of cards, each having five digits printed on it; one of the digits is a 1,2 , or 3 , and the cards have to be sorted into three piles corresponding to these digits. Four trials are given ( $T 1$, $\mathrm{T} 2$, $\mathrm{T} 3$, and $\mathrm{T} 4$ ). In $\mathrm{T} 1$, the patient is told to sort a stack of 60 cards as quickly as possible. The time taken to do this is recorded; in subsequent trials this individually determined time period is given, with the patient again required to sort as quickly as possible. T1 thus both familiarises the patient with the task and allows subsequent trial times to be adjusted to control for any sensory, motor, or cognitive deficits which reduce baseline speed.

Trials(T) 2, 3, and 4 are the experimental trials. Trials T2 and T4 are formally identical, the patient being required simply to sort the cards as quickly as possible within the specified time period. The average number of cards sorted in these trials indexes non-rewarded speed (NRSPEED). Trial T3 measures rewarded speed (REWSPEED). The patient is told that for every five cards sorted, he will receive a reward of 10 pence. During the trial, coins are placed on the table in full view after each fifth card has been sorted. Reward responsivity (REWRESP) consists in any increment in REWSPEED relative to NRSPEED - that is, REWRESP = (REWSPEED-NRSPEED).

The CARROT has been validated both in 80 normal subjects, who showed an average increase in sorting speed when rewarded of about 4\% (P<0.001; Powell and Lessiter, unpublished data), and in 54 patients with brain injury admitted consecutively to the RNRU (Al-Adawi et al, unpublished data). Within the second group, REWRESP correlated very highly with the indices of clinical motivation described above.

Within the present sample, one patient was too cognitively impaired to attempt the task at all. A second patient was physically unable to sort the cards. He therefore performed a simple finger tapping task instead of the card sorting, with the same reward and non-reward manipulation and instructions. Data were therefore available on 10 patients in total.

\section{Tests of cognitive function}

Digit span ${ }^{36}$ - This was included to index attentional span, which is likely to be partially determined by level of motivation and effort. Different number strings, randomly generated, were used on each assessment occasion. Data were available for all patients except two with severe language impairments.

Buschke selective reminding test (BSRT $\left.{ }^{37}\right)$ The BSRT was included as a test of list learning, performance on which is likely to be affected by the level of effort and strategy applied during both encoding and recall. As such it should therefore be sensitive to improvements in motivation. In the original version, there are up to 10 learning trials. However, in the present study, several subjects abandoned the test early, and the index used was therefore total score over the first three trials (completed by all subjects apart from two with severe language impairments).

For this repeated measures study, six different word lists were developed and piloted on normal subjects to ascertain that they were of equivalent difficulty (Al-Adawi, unpublished data). The word lists were given in fixed order.

Verbal fluency ${ }^{38}$ - This was included as a well established index of frontal lobe function which, unlike many other such tests, can be given in four alternate forms, thus making repeated assessment possible. The four versions were given in fixed order in consecutive assessments, and the sequence was repeated in assessments after the fourth.

The two patients with severe language deficits and one non-English speaking patient were not assessed on this measure.

\section{Mood state}

The hospital anxiety and depression scale $\left(\right.$ HADS $^{39}$ ) was given on each assessment occasion, to ascertain whether or not changes in the other indices were paralleled by alterations in anxiety and depression. These data are not available for the non-English speaking patient or for the two with severe language deficits. 
STATISTICAL ANALYSIS

Repeated measures analyses of variance (ANOVAs) were conducted for each variable, with five levels of assessment occasion (OCCASION). Each reported ANOVA was based on the subset of subjects with complete data for that variable. As there were more than two assessment occasions, Huyhn-Feldt's correction ${ }^{40}$ was applied when appropriate. In the event that there was a significant main effect of OCCASION, post hoc contrasts were used to compare scores at BL1 and BL2 with each other; MAXBROMO with BL2; and POST2 with MAXBROMO (see below for definitions of abbreviations).

\section{Results}

All patients showed a pronounced improvement in motivation at or below $10 \mathrm{mg}$ doses of bromocriptine. For two patients, the maximum dose given was $5 \mathrm{mg}$, for one it was $7.5 \mathrm{mg}$, and for eight it was $10 \mathrm{mg}$.

In the presentation of data below, scores are presented for the following occasions: the two baseline assessments (BL1 and BL2); the assessment when stabilised at maximum bromocriptine dose (MAXBROMO), which varied for individual patients between 5 and $10 \mathrm{mg}$; and the two postwithdrawal assessments (POST1 and POST2).

\section{MOTIVATION: THERAPY PARTICIPATION}

Figure 1 presents the mean \% participation index (PPI) and the motivation and spontaneity ratings for the six patients with complete data. ANOVA disclosed significant main effects of OCCASION for PPI $(F(4,20)=$ 18.4, $\quad \mathrm{P}<0.001)$, motivation $(F(4,20)=$ $13.0, \mathrm{P}<0.002)$, and spontaneity $(F(4,20)=$ $10 \cdot 0, \mathrm{P}<0.0001)$. For all three variables, post hoc contrasts confirmed that there were no significant changes across the baseline period, nor from MAXBROMO to POST1 or POST1 to POST2. However, there were highly significant increases from BL2 to MAXBROMO for all three variables (PPI: $F(1,5)=72 \cdot 7$, $\mathrm{P}<0.0001$; motivation: $F(1,5)=30.0$, P < 0.005; spontaneity: $F(1,5)=30.0, \mathrm{P}<$ 0.005 ).

Case by case inspection disclosed that every
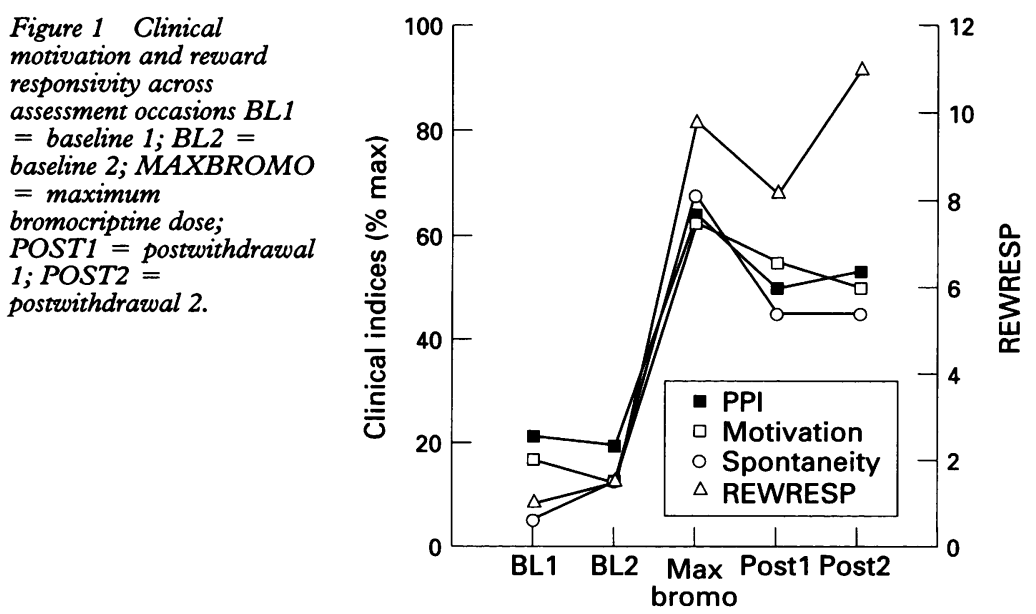

one of the eight patients on whom treatment records were available at BL2 and MAXBROMO showed improvements in PPI and spontaneity ratings after the introduction of bromocriptine; seven of eight were also given higher motivation ratings.

REWARD RESPONSIVITY: THE CARROT

Figure 1 shows REWRESP assessed for the 10 patients with complete data. The main effect of OCCASION was significant $(F(4,36)=$ $20.5, P<0.001)$. Post hoc contrasts confirmed there to be no significant change across the baseline period, but a highly significant increase after bromocriptine was introduced (BL2 to MAXBROMO: $F(1,9)=55 \cdot 3, \mathrm{P}<$ 0.0001 ); indeed, all 10 patients showed an increase in REWRESP from BL2 to MAXBROMO. After bromocriptine withdrawal, a non-significant decrease was followed by recovery to a level even higher than that achieved at MAXBROMO (POST1 to POST2: $F(1,9)=5 \cdot 4, \mathrm{P}<0.05)$.

\section{Cognitive measures}

Figure 2 presents digit span, BSRT, and verbal fluency scores. Complete data were available for nine, nine, and eight patients respectively. There were significant main effects of OCCASION for digit span $(F(4,32)$ $=10 \cdot 1, \mathrm{P}<0.001), \operatorname{BSRT}(F(4,32)=7.7, \mathrm{P}$ $<0.005)$ and verbal fluency $(F(4,28)=17.8$, $P<0.001$ )

Post hoc contrasts showed that over the baseline period verbal fluency and digit span scores remained stable, whereas BSRT scores became slightly worse $(F(1,8)=6.9, \quad P<$ $0 \cdot 05)$. For all three variables, there were highly significant improvements from BL2 to MAXBROMO (digit span: $F(91,8)=38 \cdot 3$, P $<0.001$; BSRT: $F(1,8)=12.5, \mathrm{P}<0.01$; and verbal fluency: $F(1,7)=35 \cdot 1, \mathrm{P}<0.001)$.
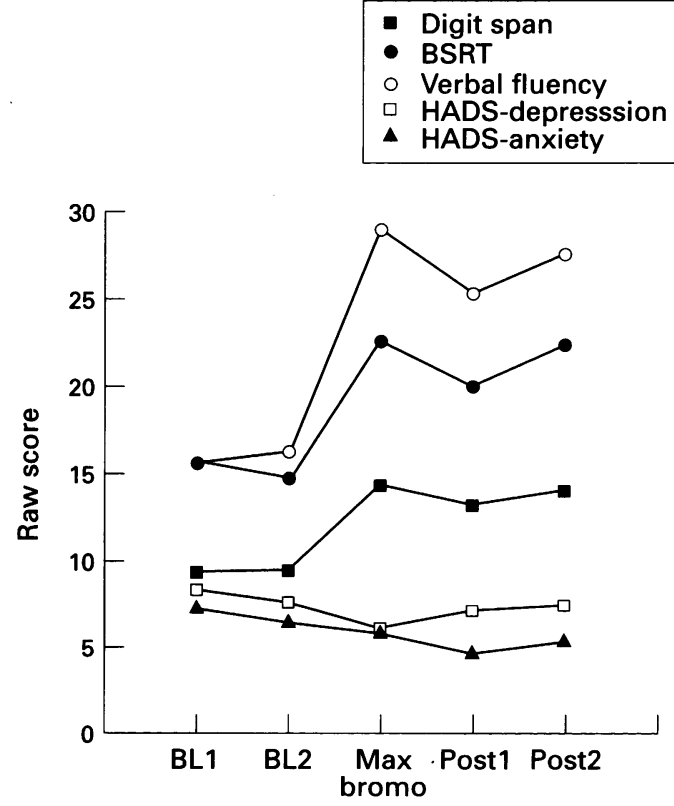

Figure 2 Cognitive test scores and mood across assessment occasions BSRT = Buschke selective reminding tests; $B L 1=$ baseline $1 ; B L 2=$ baseline 2 ; $M A X B R O M O=$ maximum bromocriptine dose; POST1 $=$ postwithdrawal $1 ;$ POST2 = postwithdrawal 2. 
There were slight, non-significant, reductions in scores on all three tests immediately after withdrawal (MAXBROMO $v$ POST1), but scores recovered to close to MAXBROMO levels by POST2. For BSRT scores, the improvement from POST1 to POST2 was significant $(F(1,8)=6.5, P<0.05)$.

All nine of the patients assessed on digit span and all eight assessed on verbal fluency showed increased scores at MAXBROMO compared with BL2. On the BSRT, seven of nine patients likewise improved after introduction of bromocriptine, whereas only one declined.

\section{Mood state}

Figure 2 shows HADS anxiety and depression scores; data were complete for eight patients. There was no significant main effect of OCCASION for either anxiety or depression $(F(4,28)=2.3$ and 1.5 respectively $)$.

\section{RE-INITIATION OF BROMOCRIPTINE}

When assessed for the second time postwithdrawal (POST2), eight patients were continuing to function at or very close to the level at which they were functioning at MAXBROMO, on most measures. Three patients, however, did show some decline after bromocriptine withdrawal. One was an outpatient whose gains while on bromocriptine were the most modest of all the patients studied. His scores on most tests fell, after withdrawal, to a point midway between his baseline and MAXBROMO levels, and it was not considered clinically appropriate to re-initiate bromocriptine. The second patient, who showed large gains while on bromocriptine and a clear reversal after withdrawal, was being treated at a different hospital and although clinical staff at that site expressed the intention to restart bromocriptine it was logistically impossible to continue with further assessments. Finally, the third patient, after making striking gains while on bromocriptine, became manifestly depressed shortly after its withdrawal. A clinical decision was made at this point to treat her with a traditional antidepressant (fluoxetine) rather than recommencing bromocriptine.

\section{Discussion}

The results of the present study are of both clinical and theoretical interest. Of 11 consecutive patients treated with bromocriptine for alleviation of abulic symptoms (for example, low motivation, poor treatment compliance, low levels of initiation, poor social interaction), all 11 seemed to respond favourably to a low dose of the drug (maximum $10 \mathrm{mg} /$ day) on a range of different indices. Eight seemed to maintain these gains when assessed on two occasions after drug withdrawal. The patients were of both sexes, with differing aetiology and loci of brain injury, and the time elapsed since the injury varied between two months and five years. It is therefore most unlikely that the changes simply reflected spontaneous recovery.

These results thus corroborate and strengthen the anecdotal reports of positive effects of dopamine agonists with similar patients reported by others. ${ }^{6-10}$ However, despite the systematicity of the methodology and assessments used in the present study, a major caveat in interpreting the findings is that the treatment was not given blind. Placebo effects are consequently possible. However, for various reasons it seems implausible that they account for the improvements in their entirety.

Firstly, the assessment measures were diverse, including both ratings by therapy staff and objective cognitive tests. The first were made across several treatment sessions by different therapists; thus although such indices may well be susceptible to the eye of faith, the striking consensus between therapists does suggest some underlying "real" improvement. More importantly, however, the perceived improvements did not reverse as staff anticipated they would when bromocriptine was withdrawn. The counter intuitive nature of this result is illustrated in one patient's discharge report: “. . . it was decided to undertake a trial of bromocriptine. In fact X did become brighter and more spontaneous but this was maintained even after the bromocriptine was discontinued so ... cannot be attributed to the bromocriptine". This reaction is not consistent with expectancy being the driving force behind the sustained improvements indexed by therapist ratings.

The cognitive tests (digit span, verbal fluency, the BSRT, and the CARROT) might potentially be influenced by both the researcher's and patients' expectations. However, the objective methods for scoring test performance in each case reduces scope for inadvertent distortion of the data by the researcher. Finally, whereas demand characteristics of the treatment may directly have motivated patients to perform more effortfully, it is notable that these patients were selected (before drug treatment) for being extremely under responsive to other forms of encouragement and explicit rewards.

It is clearly important that bromocriptine treatment should be more rigorously evaluated via a double blind, randomised, controlled trial. Pending the outcome of such a trial, however, it is relevant to consider the theoretical implications of the above data.

The results are consistent with those of a correlational study on a series of 54 consecutive patients admitted to the RNRU, conducted in parallel with the present study, in which reward responsivity on the CARROT was found to correlate extremely highly with therapy indices of motivation. In both studies, motivation and reward responsivity were largely independent of anxiety and depression, and were related to cognitive tests thought to rely on intact frontal lobe function. The data thus lend credence to the model advanced here, that motivational deficits are integrally related both to a loss of responsiveness to normal rewards and to impairments of frontal cognitive functions. It was postulated that the mechanism underlying these associations is the mesolimbic and mesocortical dopamine circuitry, activation of which is thought to be involved in the initiation, plan- 
ning, and monitoring of goal directed behaviour. Reduced efficiency in the functioning of this circuitry, arising either from focal structural damage to relevant neuronal pathways or from disruption to the synthesis, release, or metabolism of dopamine itself, would impact on the functioning of the whole system and thus have observable effects at the three levels described.

The apparent separation between impaired motivation and low mood is extremely interesting in itself, suggesting that the apathetic component of depression may be dissociable from other cognitive and somatic symptoms. A clear diagnosis of depression is often difficult in patients with brain injury, and is sometimes inferred from social withdrawal and lack of drive. If, however, some patients actually have an organic abulia with a different kind of emotional counterpart-perhaps a general "flatness" rather than distress-then a more direct boost to the dopaminergic system might be the treatment of choice.

Finally, why should the improvements in motivation and cognitive function have unexpectedly persisted in eight of the 11 patients after withdrawal of bromocriptine? On the one hand, it may be that the effects of bromocriptine outlast the half life of the drug, with readaptation taking place over a longer period than assessed here; such an effect is not uncommon with neuroleptic treatment for psychoses, in which relapse may occur after several months free of medication. Alternatively, the short, low dose treatment may effectively have "kick started" the system back into more normal, self sustaining, function. This could happen via either structural adaptations or neurobehavioural interactions in which increased behavioural output leads to increased access to rewarding activities, and these in turn stimulate dopaminergic function. It would be interesting, in future studies, to explore temporal relations between changes in behaviour, cognition, and physiological indices of central dopamine activity.

Our thanks to the therapists in the RNRU, whose active cooperation with data collection made this research possible; likewise to the staff of Northwick Park Hospital and the Royal
Hospital and Home, Putney, where two of the participating patients were treated.

1 Burgess PW, Alderman N. Rehabilitation of dyscontrol syndromes following frontal lobe damage: a cognitive neuropsychological approach. In: Wood RLI, Fussey I, eds. ropsychological approach. In: Wood RLI, Fussey I, eds.
Cognitive rehabilitation in perspective. London: Taylor and Francis, 1990.

2 Vikki J, Ahola K, Holst P, Ohman J, Servo A, Heiskanen O. Prediction of psychosocial recovery after head injury with cognitive tests and neurobehavioural ratings. F Clin Exp Neuropsychol 1994;16:325-38.

3 Levin HS, High WM, Goethe KE, et al. The neurobehavioural rating scale: assessment of the behavioural sequelae of head injury by the clinician. $\mathcal{F}$ Neurol Neurosurg Psychiatry 1987;50:183-93.

4 Ross ED, Stewart RM. Akinetic mutism from hypothalamic damage: successful treatment with dopamine agonists. Neurology 1981;31:1435-9.

5 Echiverri HC, Tatum WO, Merens TA, Coker SB. Akinetic mutism: pharmacologic probe of the dopaminergic mesencephalofrontal activating system. Pediatr Neurol 1988;4: 228-30.

6 Barrett K. Treating organic abulia with bromocriptine and lisuride: four single-case studies. $\mathcal{f}$ Neurol Neurosurg

Psychiatry 1991;54:718-21.

Parks RW, Crockett DJ, Manji HK, Ammann W. Assessment of bromocriptine intervention for the treatment of
frontal lobe syndrome: a case study. $\mathcal{F}$ Neuropsychiatry Clin Neurosci 1992;4:109-11.

8 Muller U, von Cramon Y. The therapeutic potential of bromocriptine in neuro-psychological rehabilitation of patients with acquired brain damage. Prog Neuro-
9 Nickels JL, Schneider WN, Dombovy ML, Wong TM Clinical use of amantadine in brain injury rehabilitation. Brain Inj 1994;8:709-718.

10 van Reekum R, Bayley M, Garner S, et al. N of 1 study: amantadine for the amotivational syndrome in a patient with traumatic brain injury. Brain Inj 1995;9:49-53.

11 Lindvall O, Bjorkland A, Divac I. Organization of catecholamine neurons projecting to frontal cortex in rat. cholamine neurons project
Brain Res 1978;142:1-24.

12 Robertson A, Mogenson JG. Evidence for a role for dopamine in self-stimulation of the nucleus accumbens of the rat. Can F Psychol 1978;32:67-76.

13 Bozarth MS, Wise RA. Involvement of the ventral tegmental dopamine system in opioid and psychomotor stimulant reinforcement. Life Sci 1981;28:551-5.

14 Bozarth MS, Wise RA. Heroin reward is dependent on a dopaminergic substrate. Life Sci 1981;29:1881-6.

15 Wise RA. Common neural basis for brain stimulation reward, drug reward, and food reward. In: Hoebel BG, Novin D, eds. The neural basis of feeding and reward. Proceedings from a satellite symposium of the Society for Neuroscience annual meeting, 1981. Maine: Haer Institute, 1982:445-54.

16 Fiorino DF, Coury A, Fibiger HC, Phillips AG. Electrical stimulation of reward sites in the ventral tegmental area increases dopamine transmission in the nucleus accumbens of the rat. Behav Brain Res 1993;55:131-41.

17 Yoshida $M$, Yokoo H, Mizoguchi K, et al. Eating and drinking cause increased dopamine release in the nucleus accumbens and ventral tegmental area in the rat: measurement $b$ in vivo microdialysis. Neurosci Lett 1992;139:73-6.

18 Pfaus JG, Philips AG. Role of dopamine in anticipatory and consummatory aspects of sexual behaviour in the male rat. Behav Neurosci 1991;105:727-43.

19 Wise RA. Catecholamine theories of reward: a critical review. Brain Res 1978;152:215-47.

20 Thierry AM, Tassin JP, Blanc G, Glowinski J. Selective activation of mesocortical dopaminergic system by stress. Nature 1976;263:242-4.

21 Salamone ID. The involvement of nucleus accumbens dopamine in appetitive and aversive motivation. Behav dopamine in appetitive and
Brain Res 1994;61:117-33.

22 Weddell RA, Weiser R. A double-blind cross-over placebocontrolled trial of the effects of bromocriptine on psychomotor function, cognition, and mood in de novo patients with Parkinson's disease. Behav Pharmacol 1995; 6:81-91.

23 Liddle PF. The psychomotor disorders: disorders of the supervisory mental processes. Behav Neurol 1993;6:5-14.

24 Brown AS, Gershon S. Dopamine and depression. $\mathcal{f}$ Neurol Transm 1993;91:75-109.

25 Goodwin FK, Sack RL. Central dopamine function in affective illness: evidence from precursors, enzyme inhibitors and studies of central dopamine turnover. In: Usdin E, ed. Neuropsychopharmacology of monoamines and their regulatory enzymes. New York, NY: Raven Press; 1974:261-77.

26 van Praag HM, Korf J, Lakke JPWF, Schut T. Dopamine metabolism in depressions, psychoses, and Parkinson's disease: the problem of the specificity of biological varidisease: the problem of the specificity of biological vari

27 Portin R, Rinnie UK. Neuropsychological responses of parkinsonian patients to long-term levodopa therapy. In: Rinnie UK, Klinger M, Stamm G, eds. Parkinson's disease: current progress, problems and management. Amsterdam Elsevier, 1980:270-304.

28 Burton K, Calne DB. Pharmacology of Parkinson's disease. Neurol Clin 1984;2:461-72.

29 Levi-Minzi S, Bermanzohn PC, Siris SG. Bromocriptine fo "negative" schizophrenia Comp Psychiatry 1991:32:210-6.

30 Wilner P, Muscat R, Papp M, Sampson D. Dopamine, depression and anti-depressant drugs. In: Wilner $P$, Scheel-Kruger J, eds. The mesolimbic dopamine system: from motivation to action. New York: John Wiley, 1991:387-410.

31 Blumer D, Benson DF. Personality changes with frontal and temporal lobe lesions. In: Benson DF, Blumer D, eds. temporal lobe lesions. In: Benson DF, Blumer D, eds.
Psychiatric aspects of neurologic disease. New York: Grune Psychiatric aspects of neurolo

32 Norman DA, Shallice T. Attention to action: willed and automatic control of behaviour. In: Davidson RJ, Schwartz $\mathrm{GE}$, Shapiro, D, eds. Consciousness and self-regulation: advances in research and theory, (4). New York: Plenum, 1986:1-18.

33 Thorner MO, Vance ML. Clinical aspects of dopamine in the regulation of human anterior pituitary function. In Fluckiger E, Muller EE, Thorner MO, eds. Basic and clinical aspects of neuroscience. Vol 3. The role of brain dopamine. Basel: Springer Sandoz, 1989:19-29.

34 Shakow D. Psychological deficit in schizophrenia. Behav $S_{c i}$ 1963;8:275-305

35 Richards PM, Ruff RM. Motivational effects on neuropsychological functioning: Comparison of depressed versus nondepressed individuals. $\mathcal{F}$ Consult Clin Psychol 1989;57: 396-402.

36 Wechsler D. Wechsler adult intelligence scale-revised. New York: The Psychological Corporation, 1986.

37 Buschke H, Fuld PA. Evaluating storage, retention, and retrieval in disordered memory and learning. Neurology 1974;11:1019-25.

38 Benton AL. Differential behavioural effects in frontal lobe disease. Neuropsychologia 1986;6:63-80.

39 Zigmond AS, Snaith RP. The hospital anxiety and depression scale. Acta Psychiat Scand 1983;67:361-70.

40 Huynh H, Feldt LS. Estimation of the box correction for degrees of freedom from sample data in the randomised block and split plot designs. $\mathcal{F}$ Ed Stat 1976;1:69-82. 